\title{
A NEW PROCESS FOR CROSSLINKING THERMOPLASTIC POLYURETHANES USING RUBBER INDUSTRY TECHNIQUES
}

\author{
Stéphanie LAMBOUR ${ }^{\text {a)b), }}$, Françoise MECHIN ${ }^{\text {a), }}$ \\ Jean-Pierre PASCAULT a)
}

a) Laboratoire des Matériaux Macromoléculaires, UMR CNRS 5627 Bât. Jules Verne - Institut National des Sciences Appliquées de Lyon 20, avenue Albert Einstein - 69621 VILLEURBANNE Cedex, France

b) Société d'Applications de Moulages Industriels, Route de Pinson 45330 MALESHERBES, France

Keywords: Thermoplastic Polyurethanes (TPU); processing; vulcanization; allophanates; thermomechanical behavior 


\begin{abstract}
New polyurethane materials were obtained after chemical modification of a commercial thermoplastic polyurethane (TPU) using diisocyanates and diols or diamines. The reaction can be easily processed by the well-known calendering technique often used by the rubber industry. It leads to a remarkable improvement of the thermomechanical properties of the material, due to the formation of chemical crosslinks between the TPU chains ("vulcanization"). As this new processing consists only of an intimate mixing of the starting TPU with solid reagents, followed by molding and curing stages, it allows the use of any and possibly highly reactive solid monomer (especially diamines) which could not otherwise be considered except with constraining techniques like RIM; in the same way the incorporation of organic or inorganic fillers also becomes possible.
\end{abstract}




\section{INTRODUCTION}

Polyurethane elastomers with alternating sequences of hard and soft segments make up an important class of industrial materials (1). They show excellent mechanical properties such as high tensile strength and abrasion resistance. According to the monomer reactivity, especially that of the diisocyanate and chain extender, and depending on the kind of application, different types of synthesis can be applied, starting with simple solvent-based coatings to cast-molding, reactive extrusion and finally RIM technology for the most reactive systems (2).

We propose here another way to work on polyurethanes with a technique deriving from rubber industry (3). Instead of mixing the classical reactants (polyol [polyester or polyether glycol], diisocyanate and chain extender [diol or diamine]), the new initial reactive blend consists of a preformed thermoplastic polyurethane, TPU, of reasonable molar mass (quite amorphous, or with a low melting temperature, $T_{m}$ - typically $40^{\circ} \mathrm{C}$ ) and of a diisocyanate for example. A chain extender in the solid state can also be easily added to this formulation. In this process, all solid compounds are mixed in open mills or calendered at room temperature or just below the melting temperature of each component. This constitutes the main advantage of this process, because chemical reactions occur during press curing only at high temperature. Therefore, the use of monomers with high reactivities becomes possible. Another important aspect is that this technique allows the addition of large quantities of fillers, either mineral or organic, reactive or not, a step which is usually difficult in classical processes like cast molding. 
The resulting polymer is a thermoset with a particular, slightly crosslinked structure involving allophanate or biuret bonds. In classical polyurethane synthesis, these chemical links are usually formed when using excess isocyanate with respect to hydroxyl or amine groups (4). Other side reactions (isocyanate dimerization and mainly trimerization) can also occur and change the structure and the properties of the final product $(5,6)$.

As mentioned before, the final polymer is completely insoluble and has an elastomeric behavior. Its properties are nearly equivalent to those of classical linear segmented polyurethanes with a low glass transition, a high melting point and a high storage modulus in relation with the type and proportion of hard segments formed. The stress-strain properties are also rather improved by the additional covalent bonds.

In the present work, we attempt to explain the chemical structure of such systems (TPU/diisocyanate/chain extender, possibly plus a filler), with the help of dynamic mechanical analysis to understand the final network behavior. The advantages of the process were illustrated by the preparation of materials modified with highly reactive monomers and/or fillers. 


\section{EXPERIMENTAL}

\section{Materials}

All the reagents used in this work are displayed in Figure 1. A commercial thermoplastic polyurethane (TPU) was used as precursor. Millathane ${ }^{\circledR}$ E49 (TSE industries) is a semi-crystalline polymer obtained from a polyetherpolyol $\left(\bar{M}_{n}\right.$ between 1000 and $3000 \mathrm{~g} / \mathrm{mol}$ ), extended with toluene 2,4-diisocyanate (TDI) in the presence of water to prevent any NCO chain end. The molar mass claimed by the supplier is about 10 to $15000 \mathrm{~g} / \mathrm{mol}$; a size exclusion chromatography analysis led to $\bar{M}_{\mathrm{n}}=23500 \mathrm{~g} / \mathrm{mol}$ and $\bar{M}_{w}=52100 \mathrm{~g} / \mathrm{mol}$ according to a polystyrene calibration. There are no (or very few) hard segments (HS) in this polyurethane, and thus no high melting temperature characteristic of HS. The glass transition temperature is about $-70^{\circ} \mathrm{C}$ (measured by Differential Scanning Calorimetry) followed by a crystallization exotherm at $-25^{\circ} \mathrm{C}$ and a melting

endotherm at $15^{\circ} \mathrm{C}$; which are the classical transitions for a polyoxytetramethylene chain, probably used as soft segment. This structure was confirmed by NMR, and the polymer can easily be calendered above $50^{\circ} \mathrm{C}$.

The diisocyanate used in most of the experiments is a blocked isocyanate: toluene 2,4-diisocyanate dimer or di-TDI (Thanecure T9, Rhein Chemie). Its melting point and molar mass are $156^{\circ} \mathrm{C}$ and $348 \mathrm{~g} / \mathrm{mol}$, respectively. Di-TDI has been considered with attention in this work because of its versatile functionality of either two or four if the two NCO functions masked in the uretdione ring are released. Indeed, Quérat et al. (7) demonstrated that this four-membered cycle began splitting above $150^{\circ} \mathrm{C}$ and released two new isocyanate groups. 
1,4-diisocyanatobenzene (PPDI) (DuPont) was used only for comparison, because of its poor stability in the presence of air and especially moisture. It was, however, interesting since it is supplied as a powder with a melting point higher than the calendering temperature $\left(T_{\mathrm{m}} \approx 99^{\circ} \mathrm{C}\right)$.

The chain extenders were hydroquinone-dihydroxyethylether (HQEE) (Eastman) and 4,4'-methylene bis (3-chloro-2,6-diethylaniline) (MCDEA) (Lonza) and are well known in polyurethane or polyurethane-urea fields. The diol has a melting point and a molar mass of $100^{\circ} \mathrm{C}$ and $198 \mathrm{~g} \cdot \mathrm{mol}^{-1}$, respectively. The melting point of the diamine is $98^{\circ} \mathrm{C}$ with a molar mass of $380 \mathrm{~g} \cdot \mathrm{mol}^{-1}$. Both are powders and the diamine is usually more difficult to add in classical formulations than a diol because of its higher reactivity.

Polyamide 11 (Rilsan ${ }^{\circledR}$, AtoFina) was chosen as organic filler for its high melting point, around $190^{\circ} \mathrm{C}$, which can confer better thermal properties to the polymer.

\section{Sample preparation and analytical techniques}

Polyurethane precursors and other monomers were mixed by calendering at $80^{\circ} \mathrm{C}$. Pellets or sheets of thermoplastic polyurethane were in a highly viscous liquid state during this step, with a high shear level between the rolls. The solid compounds were intimately mixed with this liquid PU. Reactions took place during press curing only at high temperature, between 120 and $150^{\circ} \mathrm{C}$, above all melting temperatures, but below the splitting temperature of di-TDI.

Size exclusion chromatography (SEC) was performed in THF using a Waters chromatograph equipped with 3 microstyragel columns (Millipore HR1, HR2 and HR3) and double detection (differential refractometry and UV absorption). When necessary, a small amount of LiCl was added in order to 
destroy hydrogen bonds (i.e. principally for E49 TPU). DSC thermograms of polyurethane rubbers were obtained by means of a METTLER TA3000 calorimeter, at a heating rate of $7.5^{\circ} \mathrm{C} / \mathrm{min}$ from -150 to $400^{\circ} \mathrm{C}$ under inert atmosphere.

The mechanical properties of these polymers depend on their complex crosslinked structure. Dynamic mechanical properties were measured using a Rheometric RSA II dynamic viscoelasticimeter with a frequency of $1 \mathrm{~Hz}$ from -120 to $220^{\circ} \mathrm{C}\left(3^{\circ} \mathrm{C} / \mathrm{min}\right)$.

Stress-strain properties were evaluated by using a tensile tester at a crosshead speed of $50 \mathrm{~mm} / \mathrm{min}$ with dumbbell type pieces at room temperature. 


\section{RESULTS AND DISCUSSION}

\section{Two-component systems (TPU/diisocyanate)}

All formulations contained a thermoplastic polyurethane, a diisocyanate, a chain extender (diol or diamine) and occasionally a filler. The TPU precursor has mostly hydroxyl and amine reactive end groups. The main possible reactions between each reagent are given below (Equations 1 to 5).

Chain extension with amine groups:

$$
\mathrm{R}^{1}-\mathrm{NCO}+(\mathrm{TPU})-\mathrm{NH}_{2} \longrightarrow \stackrel{\mathrm{O}}{\longrightarrow} \mathrm{R}^{1}-\mathrm{NH}-\mathrm{C}-\mathrm{NH}-(\mathrm{TPU})
$$

Exchange reaction with isocyanate groups:

$$
\mathrm{R}^{1}-\mathrm{NCO}+(\mathrm{TPU})-\mathrm{NH}-\stackrel{\mathrm{C}-\mathrm{O}-\mathrm{R}}{\longrightarrow} \stackrel{\stackrel{\mathrm{O}}{\|}}{\longrightarrow} \mathrm{R}^{1}-\mathrm{NH}-\mathrm{C}-\mathrm{O}-\mathrm{R}+(\mathrm{TPU})-\mathrm{NCO}
$$

Exchange reaction with alcohol groups:

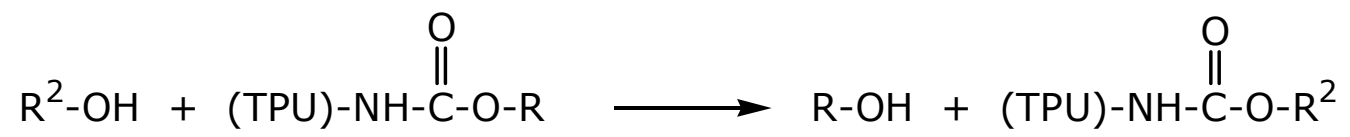

Urethane formation:

$$
\mathrm{R}^{1}-\mathrm{NCO}+\mathrm{R}^{2}-\mathrm{OH} \rightleftarrows \mathrm{R}^{1}-\mathrm{NH}-\mathrm{C}-\mathrm{O}-\mathrm{R}^{2}
$$

Allophanate formation:

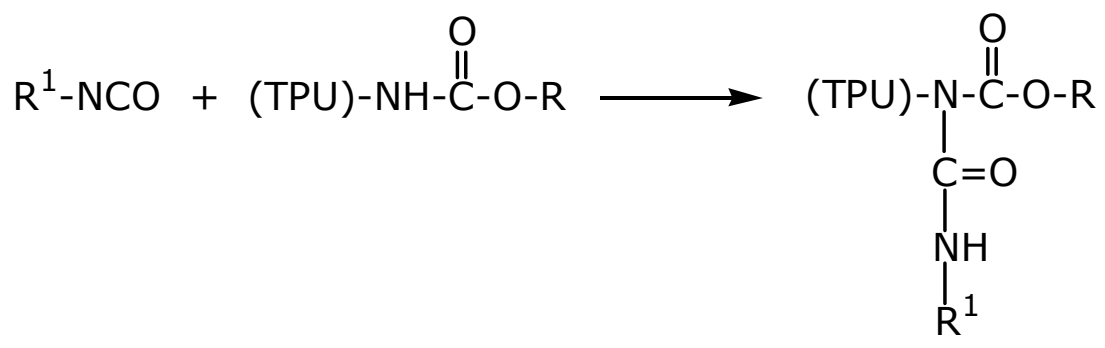


Chain extension (Eq. 1) can occur when PU chains have amine functional end groups. Exchange reactions (Eq. 2 \& 3), also known as "transurethanization" (8-12) can be observed at high temperature as a consequence of urethane scission (Eq. 4), since urethane formation is thermally reversible above $\sim 150^{\circ} \mathrm{C}$. Finally, Eq. 5 describes the main reaction involved in this process with excess NCO groups, i.e. the formation of allophanate bonds and thus of a crosslinked ("vulcanized") polyurethane.

Simple formulations were obtained at first from two-component systems, TPU and diisocyanate. The proportions used are reported in Table 1.

The degree of crosslinking can be characterized by checking the insolubility of the samples in tetrahydrofuran (THF) or dimethylformamide (DMF), if necessary with added $\mathrm{LiCl}$. Beyond a critical diisocyanate proportion, there is no soluble fraction in these formulations. In contrast, when the diisocyanate proportion is lower than this critical value, a small soluble fraction of unlinked TPU chains is observed. In the case of E49 this value falls between 7 and 8 phr; this is illustrated in Figure 2 where the size exclusion chromatograms of the THFsoluble fractions of the starting TPU and of the materials obtained with 7 or 8 phr of di-TDI are displayed. No soluble polymer chain can be detected for $8 \mathrm{phr}$, whereas the use of only 7 phr leads to the existence of a soluble fraction with a molar mass higher than the starting TPU (presumably extended or branched TPU molecules).

Referring to the above equations, one can reasonably assume that the isocyanate functions react at first with all available $\mathrm{OH}$ or $\mathrm{NH}_{2}$ groups; this can possibly lead to some chain extension. Afterwards, allophanate formation is enabled, as far as enough isocyanate groups are still available; but also exchange reactions can become non-negligible. 
The effect on dynamic mechanical properties can be seen in Figure $3 a$ and $3 b$, which show the temperature dependence of the storage modulus and of the loss factor of Millathane E49 TPU and of three formulations containing different di-TDI ratios. The starting TPU shows at first a main relaxation at $-70^{\circ} \mathrm{C}$, typical from polyoxytetramethylene soft segments, with some crystallization of these soft segments around $-40^{\circ} \mathrm{C}$, followed by their melting around $10^{\circ} \mathrm{C}$. The three modified materials show the same main relaxation around $-70^{\circ} \mathrm{C}$, with a sharp $\tan \delta$ peak at the same temperature for all the samples. The rubbery modulus holds constant till $150^{\circ} \mathrm{C}$, where a very important drop is encountered. This phenomenon can be attributed to the partial or total cleavage of the uretdione ring, allowed by the simultaneous melting of di-TDI-based crosslinks. Pure di-TDI dissociates above $150^{\circ} \mathrm{C}(7)$, whereas the splitting temperature can be higher for model diurethanes based on this molecule (13), but also sometimes lower in the presence of reactive hydroxyl groups (14). Apart from this, the fact that the sample obtained with 7 phr di-TDI was not completely crosslinked has no visible influence on this part of the curve.

All the materials are crosslinked and the rubbery plateau modulus increases as the number of crosslinks increases. More and more urethane groups are changed into allophanate links, which results in higher crosslink densities together with shorter chains between crosslinks.

In the case of the lowest diisocyanate proportion ( $7 \mathrm{phr}$ ), a shoulder is observed on the storage modulus curve after the main transition, just like for pure TPU. This can be related to some long parts of linear polyurethane chains, the amount of diisocyanate being too low to strongly crosslink the polymer and to prevent molecular motions for the soft segments. Their crystallization is thus 
enabled, in contrast to highly crosslinked networks where most soft segments have both ends tethered by allophanate crosslinks.

\section{Three-component systems: use of a chain extender}

The properties of the above materials can be further adjusted by using greater amounts of diisocyanate and by compensating for part of the isocyanate excess with a short diol or diamine (chain extender).

The networks can be depicted as in Figure 4 and 5. First, if we imagine the simple reaction between diisocyanate and polyurethane, a thermoset polymer is obtained with very few and short di-TDI segments between crosslinks (Figure 4). When the chain extender is added, all previous reactions (Eq. 1 to 5 ) can take place and lead to a different network with more distributed (-di-TDI-HQEE-) hard segments (Figure 5). Actually, HS are inserted in the "soft" network as a result of exchange reactions, and allophanate crosslinks can finally also be formed on these hard segments.

This structure can also be explained by the proportions used in the formulations. For example for $100 \mathrm{~g}$ of TPU, $30 \mathrm{phr}$ of di-TDI and $12.54 \mathrm{phr}$ of HQEE are added. 8 phr of di-TDI represents the minimal proportion to obtain a completely crosslinked material. Then, the excess isocyanate functions have multiple choices to react as shown in Eq. 1 to 5. Exchange reactions are possible, and hydroxyl functions from HQEE or urethane groups from the TPU are also potentially reactive.

Figure 6 shows typical viscoelastic spectra of three formulations containing two different chain extenders, HQEE and MCDEA, and also another diisocyanate without uretdione ring, PPDI. Different amounts of hard segments were generated in these materials; their overall compositions are indicated in Table 2 
and correspond almost exactly to the same TPU/diisocyanate/chain extender molar ratios. The formulation TPU/di-TDI/HQEE has 30 wt\% HS (calculated by the relative amount of rigid monomers, di-TDI + HQEE, in the overall material) and a rubbery modulus $\left(100^{\circ} \mathrm{C}\right)$ equal to $120 \mathrm{MPa}$ while HS proportion in TPU/diTDI/MCDEA is slightly higher, $35 \mathrm{wt} \%$ and is associated with an increased rubbery modulus, $180 \mathrm{MPa}$. This value thus strongly depends on the HS content, as it is well-known for classical linear segmented polyurethanes, polyureas and polyurethane-ureas (15-19). It can also explain why the rubbery modulus found for the material based on PPDI is so low (36 MPa), since this sample only contains 20 wt\% HS. Moreover, in the polymer based on MCDEA, the hard segments are partially crystalline and their melting point is so high that only a tiny proportion of uretdione rings can split at their usual degradation temperature; this can be a reason why, in contrast to HQEE, no sharp collapse of the modulus is observed, but rather a smooth decrease. In the last polymer with PPDI as diisocyanate, the sudden high temperature drop disappears, and is replaced by the simple melting of [PPDI/HQEE] hard segments.

For this kind of material, we noticed that the stress at break, $\sigma_{\text {break, was }}$ higher than for classical thermoplastic polyurethanes, but in return strain at break, $\varepsilon_{\text {break, }}$ was limited by additional covalent bonds. In the literature, 30 to 40 MPa are usually found for $\sigma_{\text {break, }}$ and $\varepsilon_{\text {break }}$ usually lies between 350 and $450 \%$. Here, formulations like TPU/di-TDI/HQEE or TPU/di-TDI/MCDEA show 45 and 48 MPa for $\sigma_{\text {break, }}$ and 420 and $370 \%$ for $\varepsilon_{\text {break, }}$ respectively.

This technique of combined calendering and high temperature press curing thus allows the use of highly reactive monomers, usually impossible to process by classical casting. Other diisocyanates can easily substitute for di-TDI, as long 
as they are solids at the processing temperature. In the same way, any chain extender can be introduced to control the properties of the final network.

\section{Effect of an organic filler}

Using the same process, formulations can be modified with quite high amounts of organic or mineral fillers. In this work, a linear polymer with good thermal properties (melting temperature: $190^{\circ} \mathrm{C}$ ) was added to TPU/di-TDI/HQEE. 20 wt\% TPU were replaced with polyamide 11 which represents 14 wt\% of the whole formulation (see table 3 ). All the precursors were calendered at $80^{\circ} \mathrm{C}$ and then press-cured for $4 \mathrm{~h} 30$ at $130^{\circ} \mathrm{C}$ as for previous formulations.

Figure 7 depicts the temperature dependence of storage modulus and loss factor of the filled formulation TPU/di-TDI/HQEE/PA11. The relaxation attributed to the amorphous phase of PA11 is shown in this figure by a broad $\tan \delta$ peak between 30 and $130^{\circ} \mathrm{C}$. High temperature flow begins after $190^{\circ} \mathrm{C}$, the melting temperature of PA11; which is soon followed by the melting of the polyurethane [di-TDI/HQEE] hard segments, as can be seen in the DSC curves depicted in Figure 8. The presence of these two peaks suggests that PA11 was only dispersed as a classical filler and did not react with the other components of the network. However the cure was entirely carried out below its melting temperature, and a postcure above this temperature $\left(195^{\circ} \mathrm{C}\right)$ induces a total disappearance of these melting peaks. At such a high temperature, new reactions and rearrangements may affect the TPU chains $(12,20)$, probably after HS melting; also the urethane bond reversibility can no longer be neglected. In the case of di-TDI they can further be accompanied by the release of new isocyanate functions from uretdione rings. Some reactions between amide 
groups and isocyanates have been described in the literature, and are said to evolve $\mathrm{CO}_{2}(1,21)$. In our case the material indeed gave rise to foam (density: 0.87 to $0.90 \mathrm{~g} . \mathrm{cm}^{-3}$, instead of $1.13 \mathrm{~g} . \mathrm{cm}^{-3}$ for the pure TPU/di-TDI/HQEE material).

In Figure 8, two glass transitions are also visible on the first curve that can be associated with the polyoxytetramethylene soft phase and the amorphous part of PA11, respectively. After postcuring the first one remains unchanged, while that of PA11 tends to disappear; a slight phenomenon is nevertheless visible around $110^{\circ} \mathrm{C}$. Consequently the reactions during postcuring must rather imply TPU hard segments and PA11.

\section{CONCLUSIONS}

Polyurethane elastomers synthesized classically by cast molding, extrusion or RIM technology can also be easily processed and afterwards cured by a technique well-known in the rubber field.

Curing the polyurethane chain precursors with a diisocyanate results in the formation of allophanate or biuret bonds in the polymer material. Many reactions take place between the different species and depend on the isocyanate content and processing temperature. The network properties are easily modified by the addition of a chain extender, with the creation of hard segments and exchange reactions which allow to insert them between the soft segments. The thermomechanical properties are typical of segmented polyurethanes, with a low glass transition temperature (well below $0^{\circ} \mathrm{C}$ ), a relatively high melting point depending on the type and proportion of hard segments, and a high rubbery plateau modulus (around or above $100 \mathrm{MPa}$ ). 
In this way, the design and the preparation of a broad range of new materials become possible. The calendering technique allows the incorporation of highly reactive monomers (especially amines, which usually confer better thermomechanical properties to the material but are very difficult or impossible to process other than by RIM) and of high amounts of mineral or organic fillers.

\section{ACKNOWLEDGEMENTS}

The authors wish to express their thanks to SAMI (Société d'Applications de Moulages Industriels, Malesherbes, France) for financial support and fruitful discussions. 


\section{REFERENCES}

1. J.H. Saunders, K.C. Frisch in High polymers - Polyurethanes Chemistry and Technology, Part I, John Wiley \& Sons, New York (1962).

2. C.W Macosko Fundamentals of Reaction Injection Molding, Hanser Publishers, Munich, Vienna, New York (1989).

3. A. Schroeter Rubber World 218, 30 (1998).

4. H. Nakauchi, K. Naito, S. Inoue Rubber World 214, 35 (1996).

5. T.E. Lipatova, L.A. Bakalo, A.L. Sirotinskaya Kinetica i Kataliz 21, 1246 (1980)

6. R. Arshady, M.H. George Polym. Commun. 31, 448 (1990).

7. E. Quérat, L. Tighzert, J.P. Pascault, K. Dusek Angew. Makromol. Chem. 242, 1 (1996).

8. Y. Camberlin, J.P. Pascault J. Polym. Sci. Polym. Chem. Ed. 20, 383 (1982)

9. C.D. Eisenbach, M. Baumgartner, C. Günter in Advances in Elastomer and Rubber Elasticity, Plenum Press, New York (1986).

10. J.T. Koberstein, I. Gancarz, T.C. Clarke J. Polym. Sci. Part B Polym. Phys. 24, 2487 (1986).

11. W.P. Yang, C.W. Macosko, S.T. Wellinghoff Polymer 27, 1235 (1986).

12. T. Hentschel, H. Münstedt Polymer 42, 3195 (2001).

13. P. Singh, J.L. Boivin Can. J. Chem. 40, 935 (1962).

14. R. Blum, H. Schupp Prog. Org. Coat. 18, 275 (1990).

15. C.M. Brunette, S.L. Hsu, M. Rossman, W.J. McKnight, N.S. Schneider Polym. Eng. Sci. 21, 668 (1981).

16. C. Wang, S.L. Cooper Macromolecules 16, 775 (1983). 
17. Z.S. Chen, W.P. Wang, C.W. Macosko Rubber Chem. Technol. 61, 86 (1988).

18. C.S. Wang, D.J. Kenney J. Elastomers \& Plastics 27, 182 (1995).

19. N. Samson, F. Méchin, J.P. Pascault J. Appl. Polym. Sci. 65, 2265 (1997).

20. P.J. Yoon, C.D. Han Macromolecules 33, 2171 (2000).

21. F. Parodi "Isocyanate-derived polymers" in Comprehensive Polymer Science, Vol. 5, Pergamon Press, Oxford (1989). 
Table 1: Composition of the two-component systems (TPU/di-TDI)

\begin{tabular}{|c|c|}
\hline TPU (g) & di-TDI $(\mathbf{g}) ;(\mathbf{m o l})$ \\
\hline 100 & $7 ; 2.01 .10^{-2}$ \\
100 & $8 ; 2.30 .10^{-2}$ \\
100 & $16 ; 4.60 .10^{-2}$ \\
\hline
\end{tabular}


Table 2: Composition of the three-component systems (TPU/diisocyanate/chain extender)

\begin{tabular}{|c|c|c|c|c|}
\hline Formulation & TPU $\mathbf{( g )}$ & $\begin{array}{c}\text { disocyanate } \\
\mathbf{( g )} \mathbf{( m o l )}\end{array}$ & $\begin{array}{c}\text { chain extender } \\
\mathbf{( g )} ; \mathbf{( m o l )}\end{array}$ & $\begin{array}{c}\text { hard segment } \\
\mathbf{( w t} \%)\end{array}$ \\
\hline TPU/di-TDI/HQEE & 100.00 & $30.00 ; 8.62 .10^{-2}$ & $12.54 ; 6.33 .10^{-2}$ & 30 \\
\hline TPU/di-TDI/MCDEA & 100.00 & $30.00 ; 8.62 .10^{-2}$ & $24.02 ; 6.32 .10^{-2}$ & 35 \\
\hline TPU/PPDI/HQEE & 100.00 & $13.42 ; 8.39 .10^{-2}$ & $12.26 ; 6.19 .10^{-2}$ & 20 \\
\hline
\end{tabular}


Table 3: Composition of the three-component system (TPU/di-TDI/HQEE), pure or modified by PA11

\begin{tabular}{|c|c|c|c|c|}
\hline Formulation & TPU $\mathbf{( g )}$ & $\begin{array}{c}\text { di-TDI } \\
\mathbf{( g )} ;(\mathbf{m o l})\end{array}$ & $\begin{array}{c}\text { HQEE } \\
\mathbf{( g )} ;(\mathbf{m o l})\end{array}$ & PA11 (g) \\
\hline TPU/di-TDI/HQEE & 100.00 & $30.00 ; 8.62 .10^{-2}$ & $12.54 ; 6.33 .10^{-2}$ & 0.00 \\
\hline TPU/PA11/di-TDI/HQEE & 80.00 & $30.00 ; 8.62 .10^{-2}$ & $12.54 ; 6.33 .10^{-2}$ & 20.00 \\
\hline
\end{tabular}




\section{FIGURE CAPTIONS}

Figure 1: Reagents used in this study

Figure 2: SEC chromatograms of the soluble fractions of the starting TPU and of the materials obtained by crosslinking with 7 or 8 phr di-TDI

Figure 3: Viscoelastic spectra of the starting TPU $(a)(\bullet, \diamond)$ and of three formulations TPU/di-TDI with different weight percent of diisocyanate (b) $(\bullet, 0: 7 p h r ; \Delta, \Delta: 8 p h r ; \mathbf{\bullet , ~} \square: 16 p h r ;$ dark symbols: storage modulus; clear symbols: loss factor)

Figure 4: Proposed structure for the networks based on TPU and di-TDI

Figure 5: Proposed structure for the networks based on TPU, di-TDI, and HQEE as chain extender

Figure 6: Viscoelastic properties of three networks based on TPU, diisocyanate and chain extender. $(\bullet, \diamond):$ TPU/PPDI/HQEE;

$(\Lambda, \Delta):$ TPU/di-TDI/MCDEA; $(\bullet, 0):$ TPU/di-TDI/HQEE; dark symbols: storage modulus; clear symbols: loss factor

Figure 7: Effect of 14 wt\% PA11 on the storage modulus and loss factor of TPU/di-TDI/HQEE; $(\boldsymbol{\Delta}, \Delta)$ : without PA11; $(\bullet, 0)$ : with PA11; dark symbols: storage modulus; clear symbols: loss factor

Figure 8: DSC analysis of TPU/di-TDI/HQEE formulations with or without added PA11. Effect of postcuring at $195^{\circ} \mathrm{C}$ 
Thermoplastic polyurethane (TPU):

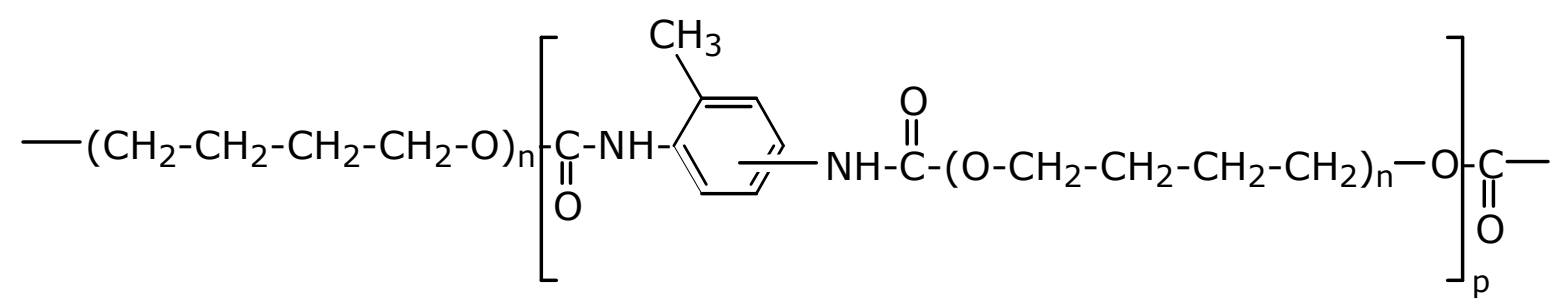
chain ends:<smiles>COCc1ccc(N)cc1</smiles>

Tolylene 2,4-diisocyanate dimer (di-TDI):<smiles>Cc1cccc(N2C(=O)N(c3cccc([N+](=O)[O-])c3)C2=O)c1</smiles>

Hydroquinone bis(2-hydroxyethyl) ether (HQEE):<smiles>OCCCOc1ccc(OCCO)cc1</smiles>

4,4'-methylene bis(3-chloro-2,6-diethylaniline) (MCDEA):<smiles>CCc1cc(Cc2cc(CC)c(N)c(CC)c2Cl)c(Cl)c(CC)c1N</smiles>

Polyamide 11 (PA11):

$$
\left[-\mathrm{NH}-\mathrm{C}-\left(-\mathrm{CH}_{2}\right)_{10}\right]_{\mathrm{n}}
$$

Figure 1: Reagents used in this study 


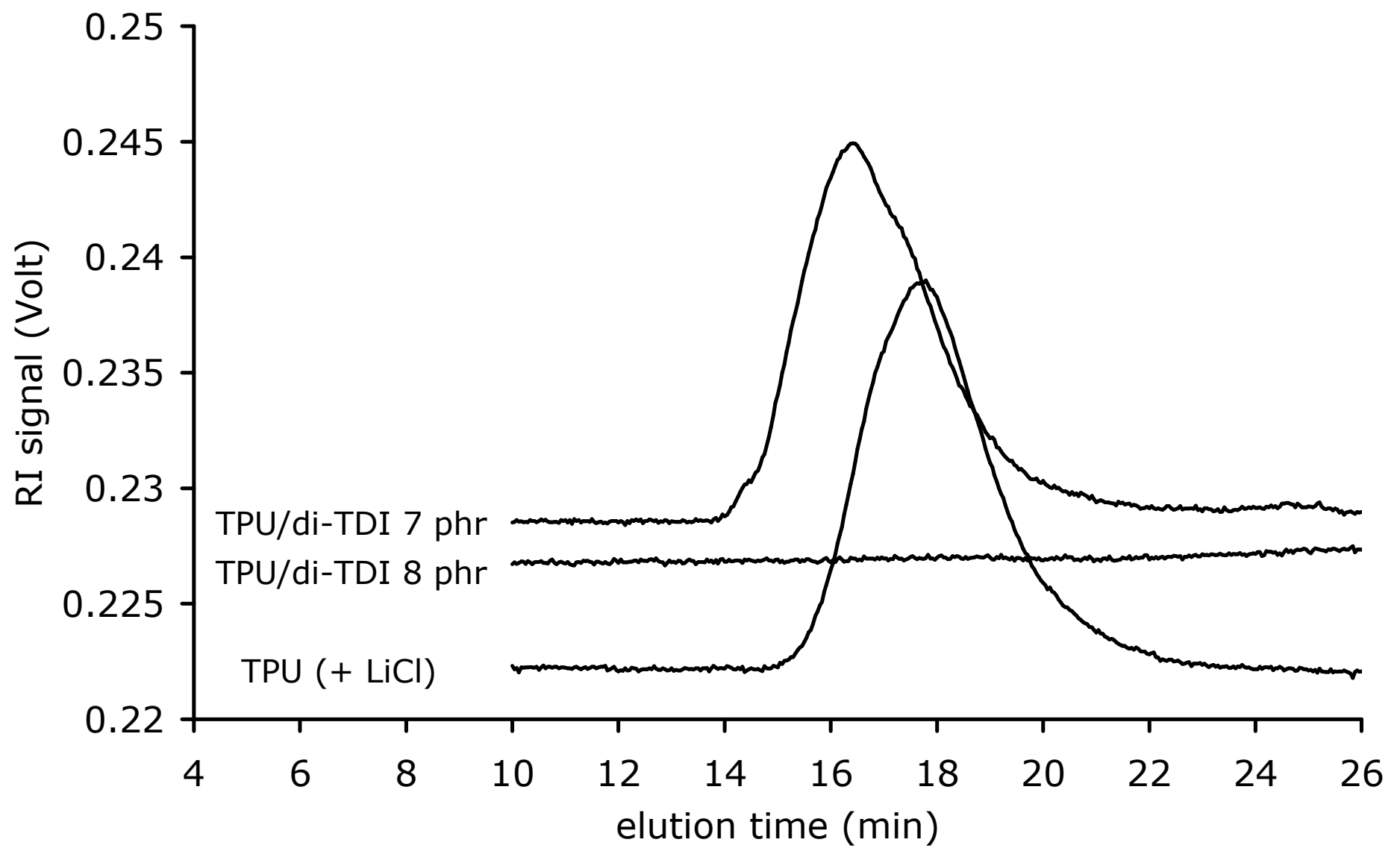

Figure 2: SEC chromatograms of the soluble fractions of the starting TPU and of the materials obtained by crosslinking with 7 or 8 phr di-TDI 


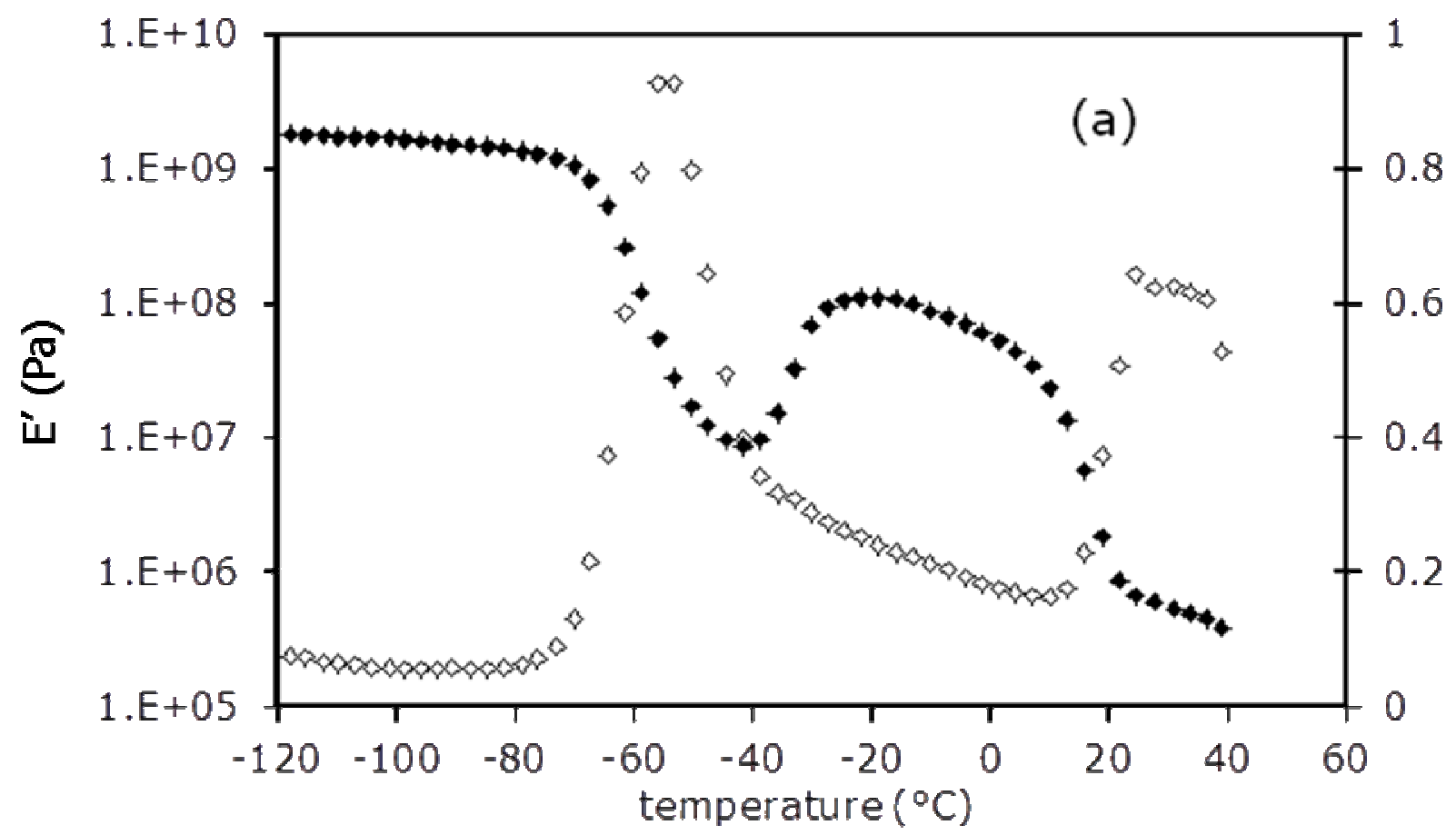

$\frac{6}{3}$

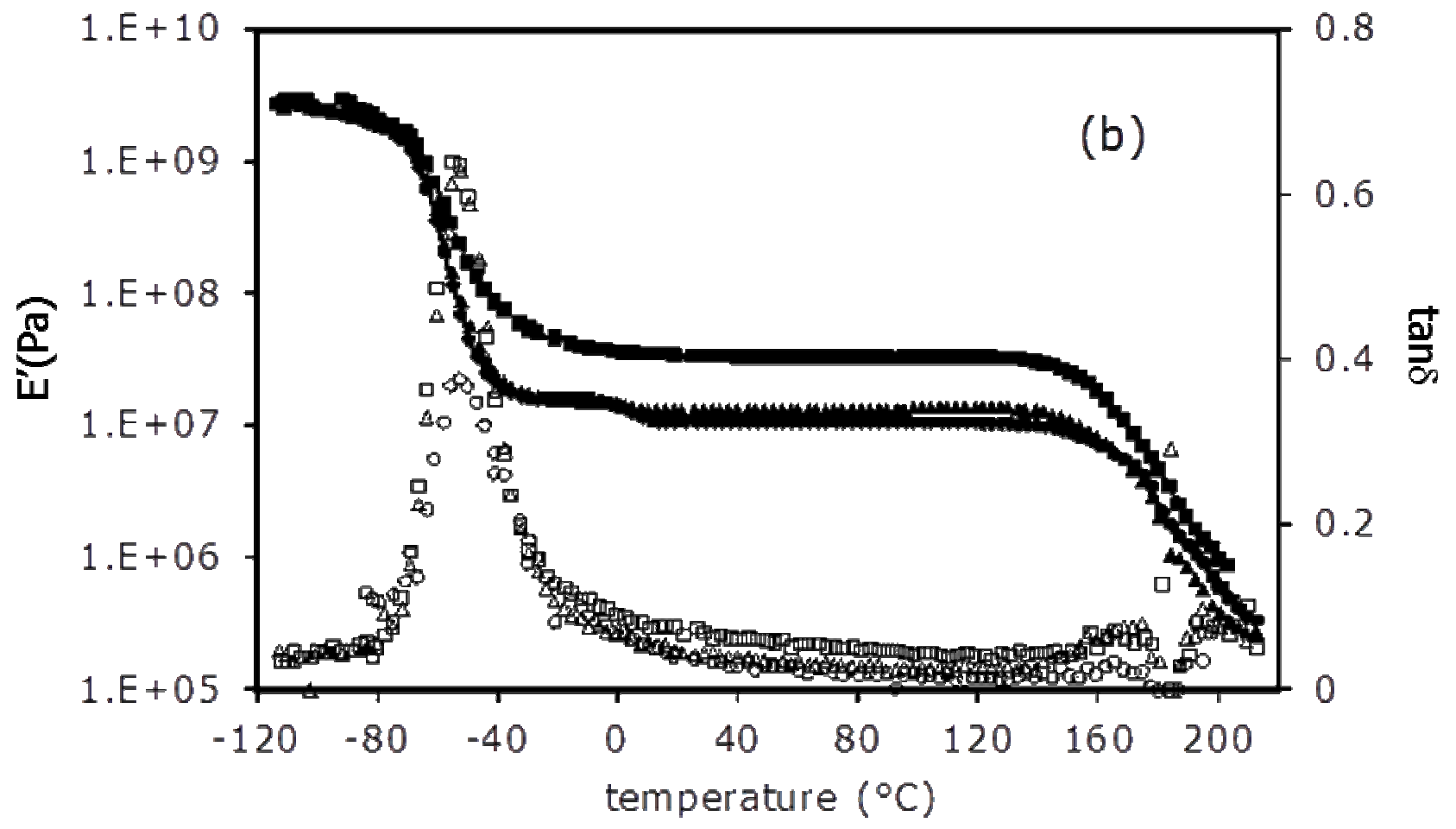

Figure 3: Viscoelastic spectra of the starting TPU (a) $(\bullet, \diamond)$ and of three formulations TPU/di-TDI with different weight percent of diisocyanate (b) $(\bullet, \mathrm{O}$ : $7 \mathrm{phr} ; \mathbf{\Lambda}, \Delta: 8 \mathrm{phr} ; \mathbf{\square}, \square: 16 \mathrm{phr}$; dark symbols: storage modulus; clear symbols: loss factor) 


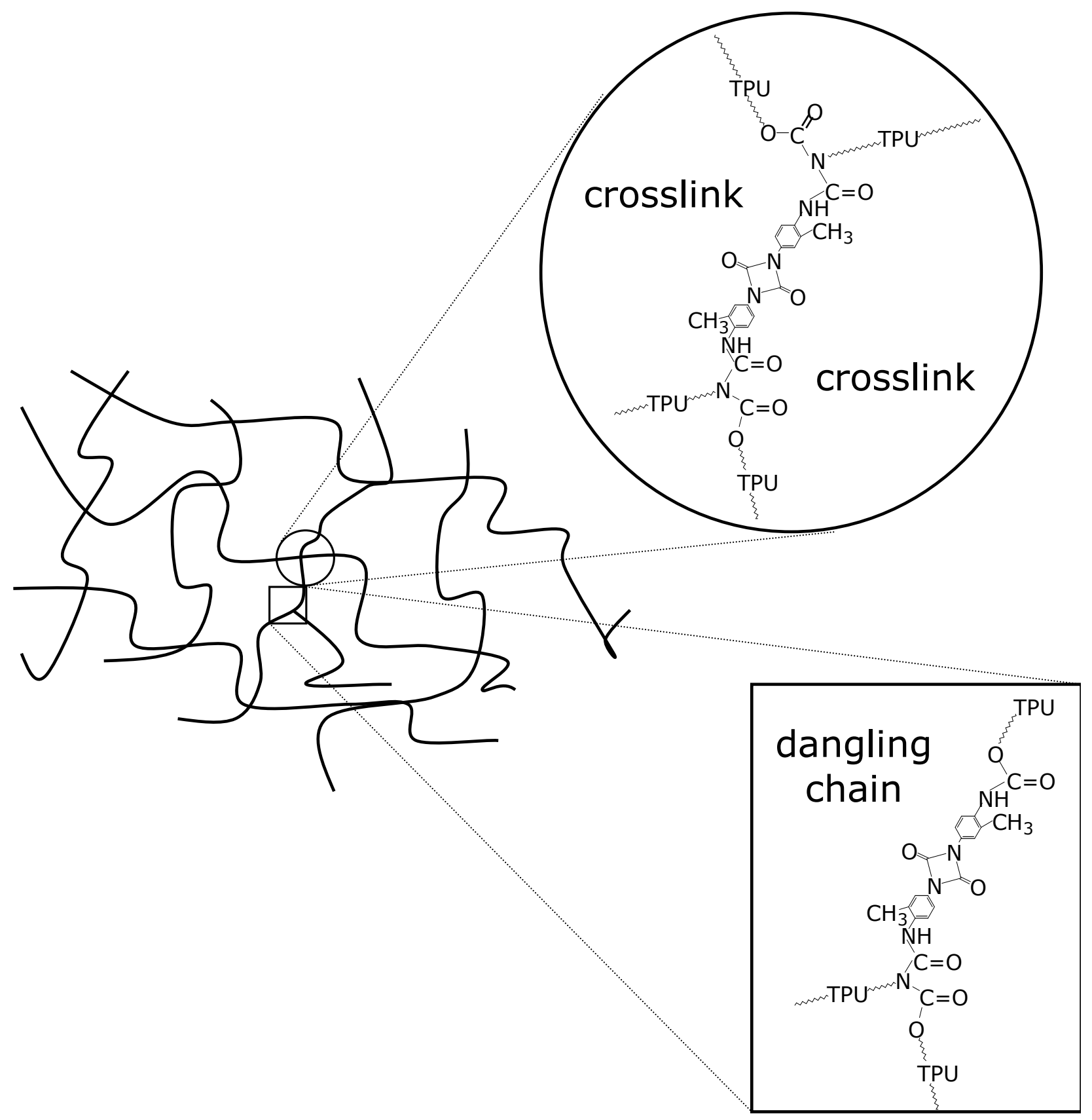

Figure 4: Proposed structure for the networks based on TPU and di-TDI 

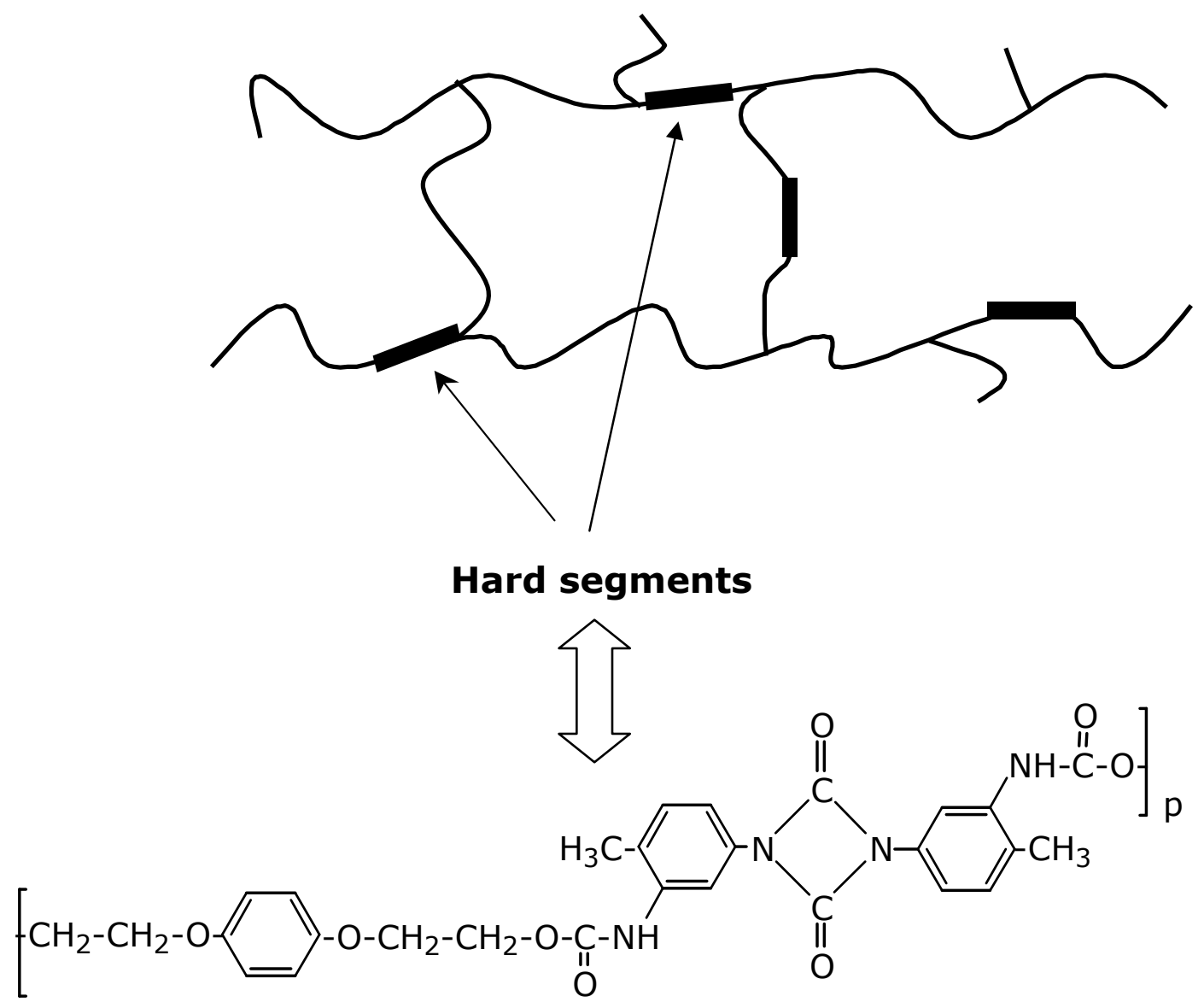

Figure 5: Proposed structure for the networks based on TPU, di-TDI, and HQEE as chain extender 


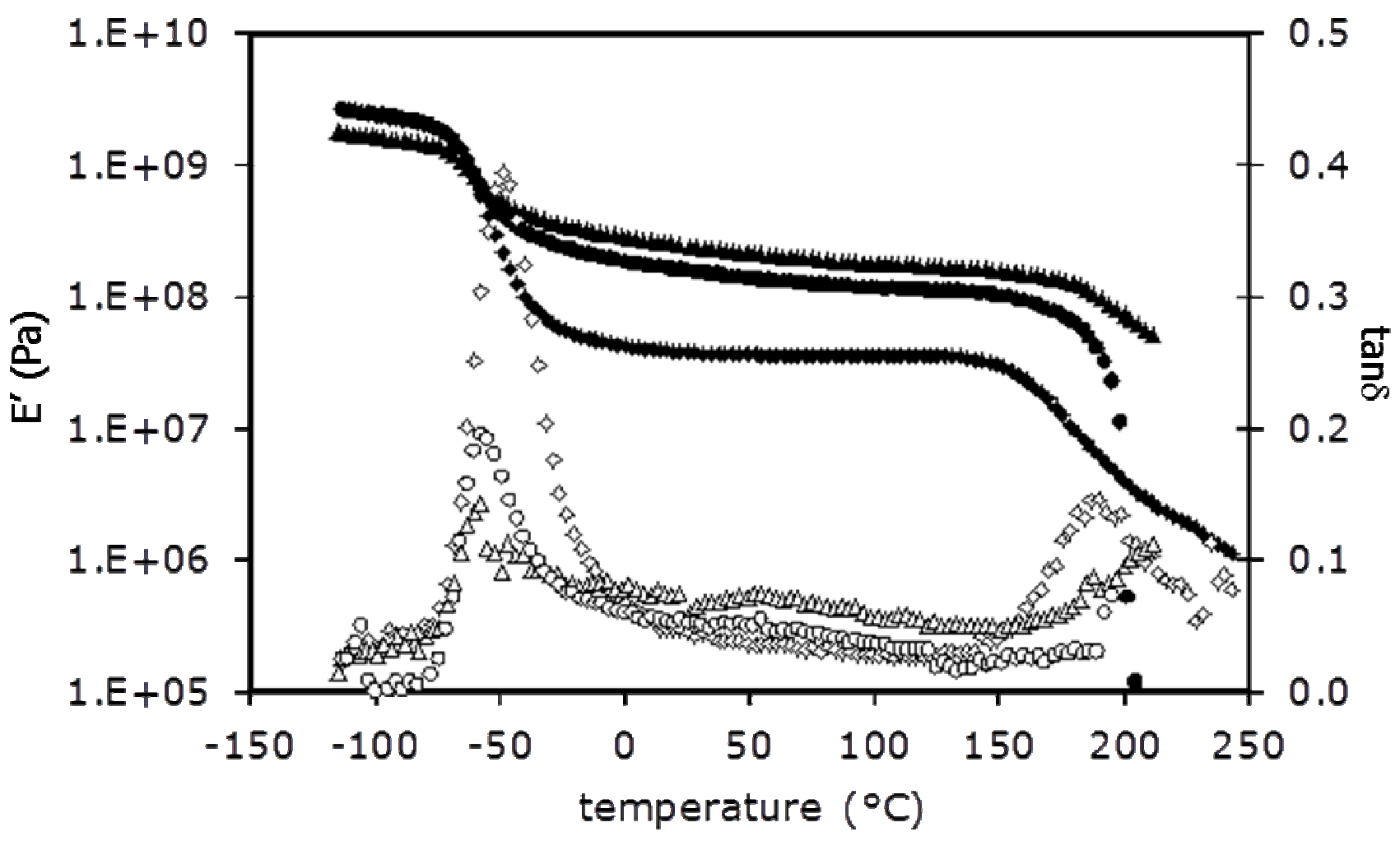

Figure 6: Viscoelastic properties of three networks based on TPU, diisocyanate and chain extender. $(\bullet, \diamond)$ : TPU/PPDI/HQEE;

$(\Lambda, \Delta):$ TPU/di-TDI/MCDEA; $(\bullet, 0):$ TPU/di-TDI/HQEE; dark symbols:

storage modulus; clear symbols: loss factor 


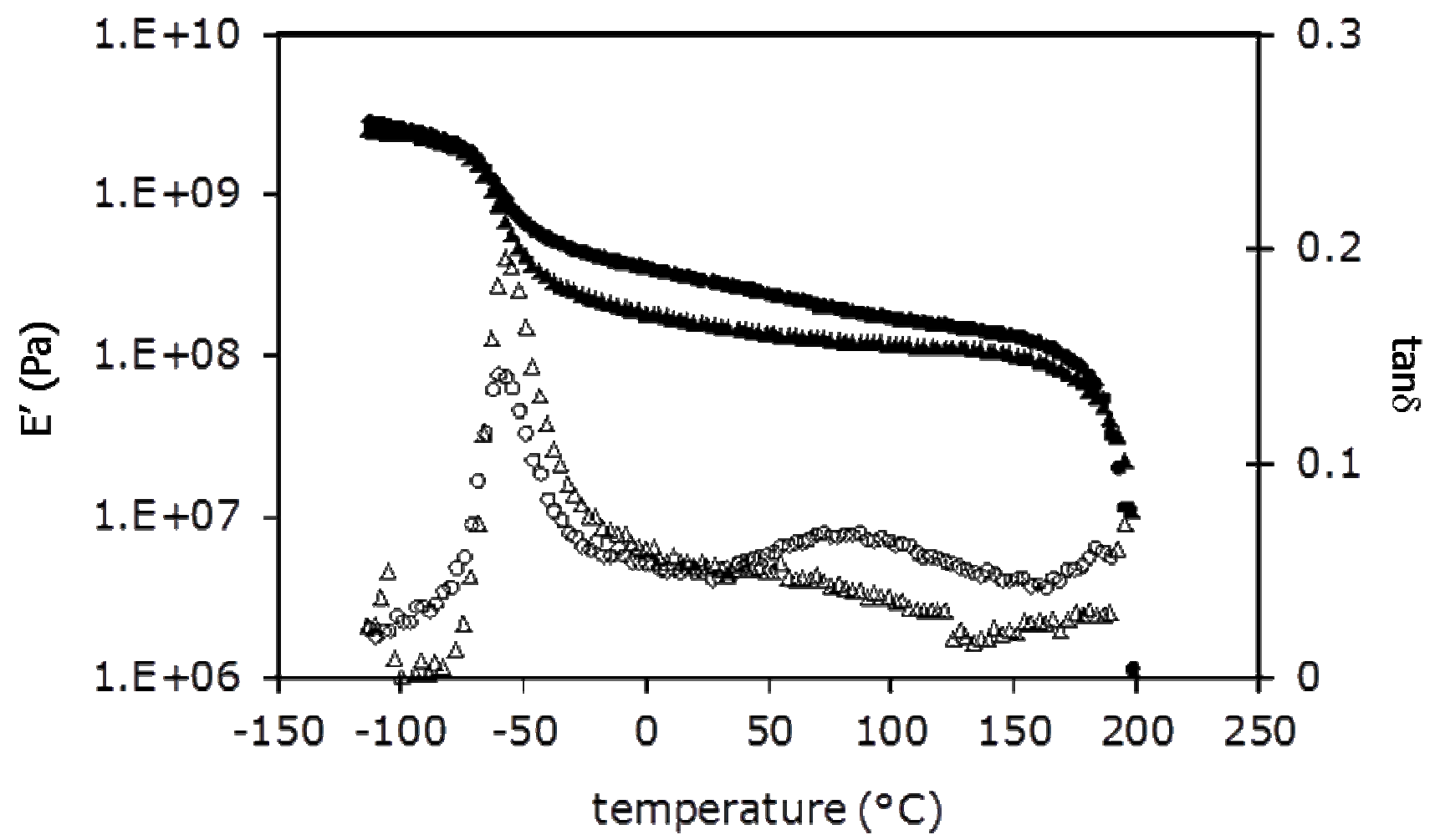

Figure 7: Effect of 14 wt\% PA11 on the storage modulus and loss factor of TPU/di-TDI/HQEE; $(\boldsymbol{\Delta}, \Delta)$ : without PA11; $(\bullet, 0)$ : with PA11; dark symbols: storage modulus; clear symbols: loss factor 


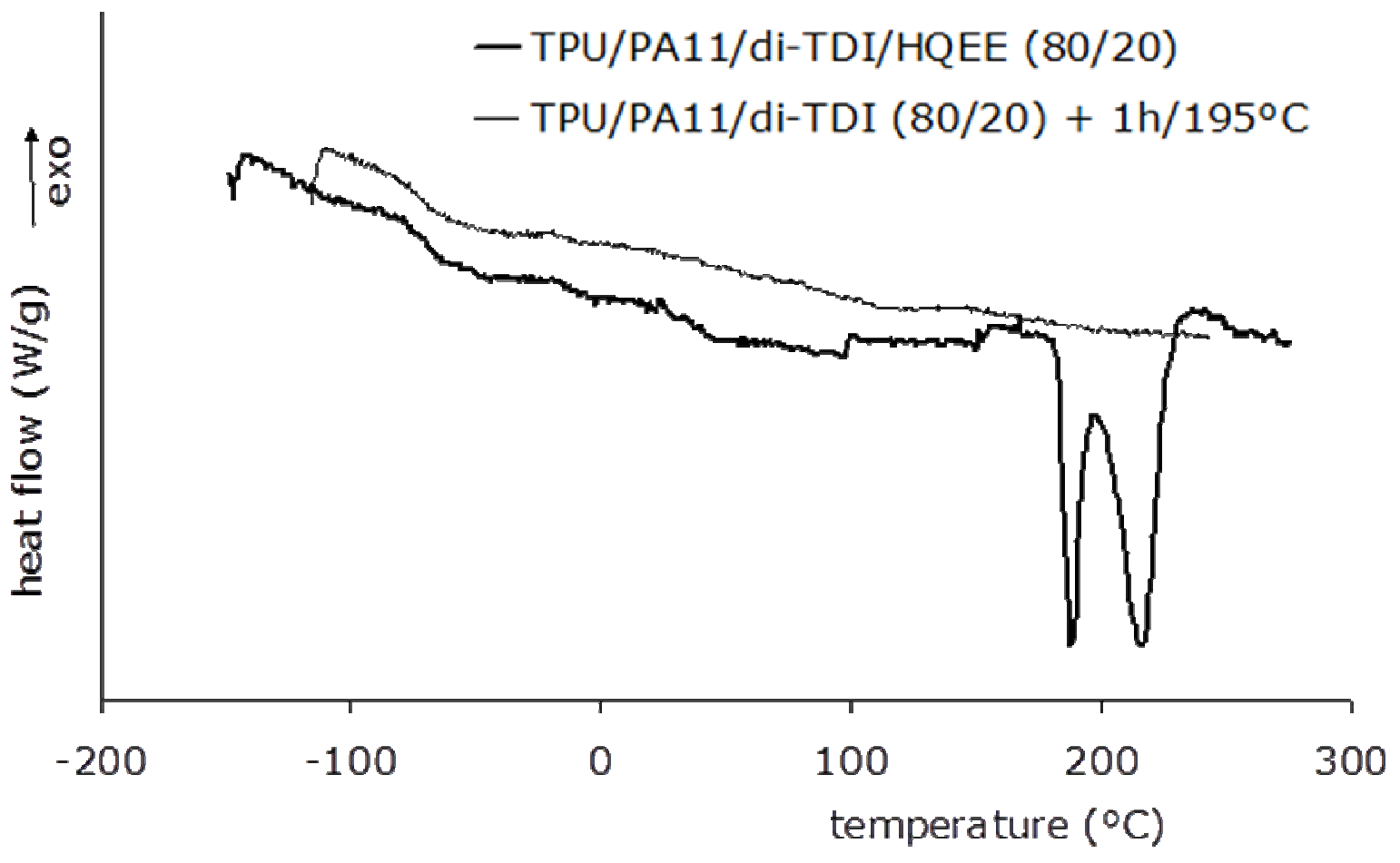

Figure 8: DSC analysis of TPU/di-TDI/HQEE formulations with or without added PA11. Effect of postcuring at $195^{\circ} \mathrm{C}$ 